\title{
REVISIONES
}

\section{Relaciones de poder en la instalación de la reforma educativa en Chile}

\author{
Power relationships in the placing of the Educational Reform in Chile
}

Relações de poder na instalação da Reforma Educativa no Chile

\author{
Leonardo Vidal Araya \\ Universidad ARCIS, Escuela Latinoamericana de Estudios de Postgrado, Programa de Doctorado en Cultura \\ y Educación en América Latina, Libertad 53, Santiago de Chile, leonardovidalaraya@gmail.com
}

\begin{abstract}
RESUMEN
El autor realiza una aproximación a la instalación de la reforma educativa en Chile a través del eventual campo de fuerzas que constituye la trama de relaciones de poder inherentes al sistema educativo. Desarrolla una aproximación analítica mediante una focalización selectiva de algunos hechos o situaciones que permitirían obtener una cierta caracterización de un campo muy dinámico y complejo. Finalmente, se establece como conclusión principal que en la instalación de la reforma educativa, los niveles políticos y técnicos no habrían considerado la situación estratégica y el potencial de ciertos comportamientos, relaciones de poder y resistencias que, de modo a veces silencioso, estarían afectando los equilibrios o desequilibrios en el campo de fuerzas del sistema educativo y, en alguna medida, incidiendo en la efectividad de las políticas educativas.
\end{abstract}

Palabras clave: reforma educativa, relaciones de poder, políticas educativas.

\begin{abstract}
The author presents a rapprochement to the placing of the educational reform in Chile through the field of forces which constitutes the interweaving of power relations within the educational system. He develops an analytical approach by selectively focusing on some facts or situations which allow a sort of characterization of a very dynamic and complex field. Finally, as a main conclusion, it is stated that in the installation of the educational reform, the political and technical powers would not have considered the strategic situation and the potential of certain behaviors, relations of power and resistances that, sometimes, in a silent way, would affect the balances or imbalances in the field of forces of the educational system and, to some extent, affect the efficiency of the educational policies.
\end{abstract}

Key words: educational reform, relations of power, educational policies.

\section{RESUMO}

Apresenta-se aproximação da Reforma Educacional no Chile, por meio de possível campo de forças que constituem relações de poder inerentes ao sistema educacional. Desenvolve-se aproximação analítica, mediante a seleção de alguns acontecimentos ou situações que permitiram caracterizar um campo bastante dinâmico e complexo. Finalmente, conclui-se que o desenvolvimento da Reforma Educativa, em seus níveis políticos e técnicos não considerou a situação estratégica e o potencial de certos comportamentos, relações de poder e certas resistências que, silenciosamente, estão prejudicando o (des)equilíbrio no campo de força do Sistema de Ensino. De certa forma. Isso tem afetado a eficácia das políticas educacionais.

Palavras chave: reforma educacional, relações de poder, políticas educacionais. 


\section{INTRODUCCIÓN}

Las Reformas Educativas se generan, instalan y permanecen en una compleja dinámica de relaciones de poder en la que se entremezclan diversas entidades y actores sociales, nacionales e internacionales. Esta compleja trama de interacciones y antagonismos de fuerzas sostiene y dinamiza la macro y micropolítica del sector educativo. Así las fuerzas resultantes posicionan un campo de acción relativo de avances, estancamientos o retrocesos que constituye la actual realidad de la educación chilena.

La noción de "relaciones de poder" que se adopta en este trabajo se corresponde con la perspectiva foucaultiana asociada al poder que pone en juego las relaciones entre individuos o grupos. Foucault establece una diferencia entre capacidades objetivas, relacionamientos comunicacionales y relaciones de poder; aunque éstos no constituyen dominios separados, conforman un entramado de relacionamientos superpuestos que se mantienen y utilizan para determinados fines. A diferencia de la relación de violencia que actúa sobre un cuerpo o cosas para forzar, doblegar o destruir, "lo que define una relación de poder es que es un modo de acción que no actúa de manera directa e inmediata sobre los otros, sino que actúa sobre sus acciones: una acción sobre la acción, sobre acciones eventuales o actuales, presentes o futuras" (Foucault, 1988: 14).

En los niveles de macro decisiones el campo de fuerzas estaría controlado y regulado por el poder económico y el poder político, con la participación del estamento intelectual. En el nivel de decisiones técnicas las relaciones de poder se darían principalmente entre los diversos actores y entidades del ámbito de la gestión administrativa y curricular del sistema escolar. En un nivel operativo, se encuentran los principales actores del proceso educativo: alumnos, docentes, directivos, padres o apoderados, los que están en el contexto de acción directa de la organización escolar o establecimiento educacional.

A través del presente trabajo se pretende realizar una aproximación -que no tiene la pretensión de ser exhaustiva- a la instalación de la reforma educativa en Chile ${ }^{1}$, a través del eventual campo de fuerzas que constituye la trama de relaciones de poder inherentes al sistema educativo. La aproximación y el análisis se realizan mediante una focalización selectiva de algunos hechos o situaciones que permitirían obtener una cierta caracterización de un campo muy dinámico y complejo. En esta compleja red de interacciones, inmersa en el sistema social, las relaciones de fuerza se manifiestan en diferentes sentidos y magnitudes, permeando todos los niveles del sistema educativo. Sin embargo, con un propósito analítico, en primer lugar nos aproximaremos a la dinámica de poderes políticos y económicos, para luego analizar las relaciones de poder en el ámbito de la gestión educativa. Finalmente se analizará la eventual trama de relaciones de poder en el nivel operativo, focalizando la atención en los docentes como principales agentes del proceso educativo. La decisión de dejar el nivel operativo para el final, no significa necesariamente que estos actores sean los más débiles en términos del ejercicio de poder, en consideración a la noción de poder que se ha adoptado en el presente trabajo.

1 El análisis se realiza a partir de la reforma del 80 con cierto énfasis en la reforma de los años 90. 


\section{LA INFLUENCIA DE LA ECONOMÍA}

El rol protagónico de la economía en las relaciones de poder en el contexto social alcanza directamente al ámbito educativo, se imprime un sello de direccionamiento vertical y se expanden transversalmente sus efectos en los diferentes estamentos, instancias, organismos e instituciones. En la década de los ochenta se expanden en América Latina las ideas y reformas neoliberales, las que se introducen y materializan en el ámbito educativo a través de reformas y políticas educativas. Un importante hito en la historia educativa reciente de Latinoamérica lo constituye la Conferencia Regional de Ministros de Educación y de Ministros Encargados de Planificación Económica, celebrada en México en diciembre de 1979. Allí se establecieron importantes declaraciones y llamamientos a los países miembros que quedaron plasmados en el documento que se conoce con el nombre de "Declaración de México". ${ }^{2}$. La gran mayoría de las declaraciones establecidas en ese documento se refieren al establecimiento de algunas premisas o supuestos fundamentales en los cuales se manifiesta de manera explícita una estrecha y vinculante relación entre educación y desarrollo en la región. A partir de la Declaración de México se da inicio a un proceso de importantes cambios en los sistemas educativos.

La estrecha vinculación que se establece entre Educación y Economía resulta absolutamente obvia y esperable desde la génesis de la conferencia, en la que participaron los Ministros de Educación y los Ministros Encargados de la Planificación Económica. Particularmente en una de las declaraciones se deja de manifiesto el carácter prioritario y consensual respecto de una economía internacional: "que es necesario el establecimiento de un nuevo orden económico internacional como prerrequisito básico para que los países de la región puedan llevar a cabo sus proyectos nacionales." En la Declaración de México también se establecen una serie de acciones específicas en materia educativa que deberían acometer los Estados Miembros, entre las cuales está la instrucción específica de "emprender las reformas necesarias para que la educación responda a las características, necesidades, aspiraciones y valores culturales de cada pueblo" (cursiva mía), y la afirmación de que los Estados deberían "concebir el crecimiento económico dentro de un amplio contexto de desarrollo social, vinculando estrechamente la planificación de la educación con la planificación económica, social y global del país."

La declaración de México constituye un texto en el que se plasma con sorprendente nitidez la notable influencia de la Economía sobre la Educación. Las fuerzas de la economía desde la década del ochenta van a ejercer un abierto e indisimulado direccionamiento de la Educación en Latinoamérica y El Caribe. En la misma conferencia de México también se hace un llamamiento a la UNESCO para que tome la iniciativa de proponer un proyecto principal que incluya los elementos fundamentales incluidos en la Declaración de México. En respuesta a esa solicitud, la Conferencia General de la UNESCO en su 21 a reunión, realizada en Belgrado, septiembre-octubre de 1980, autoriza al Director General a Convocar la Reunión Regional Intergubernamental, en abril de 1981 en la ciudad de Quito sobre los Objetivos, las Estrategias y las Modalidades de Acción de un Proyecto Principal en la Esfera de la Educación en la Región de América Latina y el Caribe.

2 Declaración de México: aprobada por aclamación por la Conferencia Regional de Ministros de Educación y Ministros Encargados de la Planificación Económica de los Estados Miembros de América Latina y del Caribe, organizada por la UNESCO con la cooperación de la CEPAL y de la OEA. 
El Proyecto Principal de Educación de América Latina y el Caribe marcó el inicio del desarrollo educativo de la década de los 80 . En ese proyecto se introdujo explícitamente la noción de calidad a través de uno de sus objetivos y se llamó a los países de la región a emprender las reformas necesarias con el propósitos mejorar la calidad y la eficiencia de los sistemas educativos. La década de los 80 se caracterizó por una época de crisis estructural, lo que sin duda tuvo repercusión en el desarrollo educativo de la región. Se produjo a una transición a modelos democráticos en situación de inestabilidad con serios problemas de deuda externa, dificultades para el acceso a créditos y modelos de producción que no respondían a las nuevas exigencias económicas y laborales. El contexto histórico de la década de los ochenta resultó especialmente propicio para la manifestación en plenitud de los poderes de la economía sobre el ámbito educativo. En ese contexto adverso, el Proyecto Principal de Educación se presenta como una forma de respuesta a los problemas y necesidades de un período crítico, lo que favorece la aceptación por parte de los Estados Miembros de América Latina y del Caribe y potencia una influencia activa y efectiva del poder de la economía en los sistemas educativos durante los años siguientes.

Según Castells, desde la década del 80, Chile transita de un modelo de desarrollo autoritario liberal excluyente a un modelo democrático liberal incluyente. "El calificativo de liberal se funda en el énfasis que ambos modelos ponen en el mercado y en la apertura económica internacional como elementos esenciales del crecimiento económico" (Castells, 2005: 58). Estos dos modelos de desarrollo que describe Castells tendrían su correlato en el sistema educativo chileno, en la reforma pro mercado de la década del ochenta y el modelo de integración de Estado y mercado en las políticas educacionales y de reforma de los noventa. No obstante, como afirma Castells, ambos modelos mantienen los énfasis en el mercado y en la apertura económica internacional como ejes del crecimiento económico. "El nuevo modelo escolar y educativo que tiende a imponerse está fundado, en primer lugar, en el sometimiento más directo de la escuela a la razón económica" (Laval, 2005: 68). De manera que la reforma de los noventa, aunque incorpora cambios respecto de una nueva relación de integración de Estado y mercado, se mantiene fiel a la estrecha vinculación entre la planificación de la educación y la planificación económica del país. En definitiva la reforma educativa chilena se mantiene alineada con el direccionamiento y coordinación de la UNESCO, a través del Proyecto Principal de Educación para América Latina y el Caribe, iniciado en la década del 80 (1980-2000), lo que se va a traducir en la práctica en importantes ejes de continuidad respecto de la reforma de los ochenta.

La influencia de la economía en el ámbito educativo es cada vez más amplia y profunda. Al respecto Casassus plantea: "Sin llegar a decir que las políticas educativas tienen un fin económico explícito, sí se puede afirmar que la orientación valórica de las políticas ha estado dominada por los conceptos económicos" (Casassus, 2005: 155). Esta abrumadora influencia de la economía desde la génesis de las políticas educativas hasta el nivel operativo de la administración escolar, encargada de la implementación de las políticas en el nivel de los agentes educativos y beneficiarios, se traduce en una situación estratégica que muchas veces resulta desequilibrante en las relaciones de poder entre los diferentes actores del sistema educativo. 


\section{EL PODER POLÍTICO}

Las relaciones de poder en política y economía se dan en un entramado de acciones, interacciones e influencias recíprocas. En los Estados, la política y la economía actúan concomitantemente y afectan de diversas formas las relaciones entre las personas, grupos e instituciones. De manera que sería inapropiado o artificial afirmar la existencia una clara distinción y separación entre el "poder político" y el "poder económico". Según Foucault "el poder no es una institución, y no es una estructura, no es cierta potencia de la que algunos estarían dotados: es el nombre que se presta a una situación estratégica compleja en una sociedad dada" (Foucault 1991: 113).

El modelo de desarrollo autoritario liberal excluyente impulsado por el gobierno militar fue respaldado por un ejercicio autoritario del poder del Estado. Se caracterizó por priorizar los mecanismos de mercado por sobre los valores de solidaridad social. Las elites sociales y económicas gozaron de privilegios, lo que provocó grandes desigualdades y significó la exclusión de los beneficios del crecimiento para gran parte de la población. En ese contexto político económico de la década del 80, el régimen político autoritario tenía la sensibilidad ideológica, el poder y las facultades extraordinarias que le permitieron alinearse con la declaración de México y responder plenamente a sus llamamientos, específicamente a la instrucción de emprender una reforma educativa, dentro de una concepción de desarrollo social que vincula la planificación de la educación con la planificación económica del país. Así se inicia un proceso de reforma estructural que involucra profundas transformaciones en el modelo de financiamiento y de gestión del sistema escolar (Cox, 2003). Se descentralizó la administración, se introdujo instrumentos de financiamiento basados en el subsidio a la demanda y, mediante instrumentos legales e incentivos de mercado, se estimula la iniciativa privada para la creación de establecimientos educacionales con financiamiento del Estado.

En las políticas descentralizadoras y privatizadoras de los años 80 se expresan algunos propósitos gubernamentales explícitos e implícitos:

i) el logro de una mayor eficiencia en el uso de los recursos a través de una competencia entre establecimientos por matrícula; ii) el traspaso de funciones desde el Ministerio de Educación y su burocracia central a los poderes locales representados en el municipio; iii) la disminución del poder de negociación del gremio docente; iv) una mayor participación del sector privado en la provisión de la educación; y v) una cercanía mayor de la educación media técnico-profesional a los ámbitos económicos de la producción y los servicios (Cox, 2003: 23).

Estos propósitos materializados en la reforma educativa de los 80, en la práctica establecen una particular configuración del campo de relaciones de poder en el sistema educativo chileno. En opinión del mismo Cox (2003:24), "la reforma de comienzos de la década de 1980 tuvo fundamentos financieros, de eficiencia y de distribución de poder, los que se interpretaron como decisivos para una mejora de la calidad de la Educación." Esta compleja dinámica de relaciones de poder va a conformar y caracterizar el desarrollo del sistema educativo en la década del ochenta hasta el advenimiento del gobierno democrático, tiempo en el cual se produce una suerte de perturbación sistémica que altera en alguna medida las situaciones estratégicas de poder en el sector educativo.

Bajo el Estado democrático surge el modelo democrático liberal incluyente, en la década del 90. Aunque en este modelo se mantiene los mecanismos de mercado como 
forma de asignación de recursos, se implementan políticas públicas destinadas a incluir el conjunto de la población en los beneficios del crecimiento. Además se elaboran políticas públicas a través de mecanismos de consulta y negociación con los grupos de intereses y diversos actores sociales, orientados a la búsqueda de consenso través de sus representantes (Castells, 2005). Las políticas educativas del gobierno de la concertación de partidos por la democracia incorporan un cambio importante respecto del rol del Estado. El rol esencialmente subsidiario se abre hacia un rol promotor. El Estado se encarga de definir y conducir las políticas de desarrollo del sector educativo e inicia un período de intensa actividad con un crecimiento significativo del gasto en educación. Una de las principales preocupaciones de la agenda educativa se relaciona con la promoción de objetivos de calidad y equidad del sistema educativo, respecto del contexto y de los resultados de aprendizaje obtenidos por los alumnos.

La reforma de los noventa trae consigo una reconfiguración del campo de relaciones de poder, aunque se mantiene la situación estratégica de poderes asociados a algunas instancias, estamentos o instituciones. Se mantienen los rasgos estructurales de la reforma educativa de los años 80, impulsada por el gobierno militar, específicamente los componentes de organización y financiamiento del sistema. El gobierno democrático estratégicamente decide mantener el sistema de municipalización, el modelo de financiamiento basado en la subvención por alumno y el papel del sector privado en educación. También se continúa con el sistema de medición de logros de aprendizaje. Es importante destacar que el gobierno democrático tomó esa decisión a pesar del origen autoritario de los cambios implementados en la reforma del ochenta y en contra de las expectativas que tenía el profesorado respecto de esas materias (Cox, 2003). No obstante, el gobierno establece algunos ejes de ruptura con las políticas educativas implementadas por el gobierno militar. El papel de la educación pasa de un nivel de prescindencia a tener una función estratégica en el proyecto país, expresado en un crecimiento sostenido que experimenta el gasto en educación en la década del 90. Además se redefine el status de la profesión docente, devolviéndole una condición de protegida públicamente y el Colegio de Profesores se constituye en la organización nacional que se relaciona centralizadamente con el Estado.

En la primera década del 2000 prácticamente no ha habido diferencias sustantivas respecto de políticas educativas. Un hecho relevante fue la movilización de estudiantes de educación media del 2006, conocida como la revolución de los pingüinos, quienes exigían, entre otras cosas, la derogación de la Ley Orgánica Constitucional de Enseñanza (LOCE). Este acontecimiento dio lugar a un convulsionado proceso de tramitación de una Ley General de Educación (LGE), que originó oposición en el gremio docente y en los propios alumnos. Sin embargo, el Proyecto de Ley siguió su curso respaldado en un nivel de consenso logrado entre el gobierno y la oposición durante el periodo de la Presidenta Michelle Bachelet. En los primeros meses del 2010, con la llegada de un gobierno de derecha, parlamentarios de la concertación, antes gobierno y actualmente oposición, han manifestado su rechazo a algunos artículos fundamentales del proyecto. Son aspectos de especial controversia la creación de una agencia de calidad y una Superintendencia de Educación.

El proyecto de Ley General de Educación tiene como foco la "calidad de la educación", no obstante, se trata de una noción ambigua que no está claramente definida. Lo que es más claro, como afirma Casassus, es que "calidad no está referida a un elemento abstracto y trascendente, sino una cuestión relativa a personas y grupos y, por lo tanto, 
sujeta a relaciones de poder" (2006: 3). Casassus crítica también la lógica empresarial que está presente en el proyecto de Ley General de Educación y plantea que en los últimos 25 a 30 años la educación ha tenido un giro ideológico que no ha contribuido precisamente a mejorarla. El ethos empresarial ha reemplazado al ethos humanista. "Chile ha sido exitoso en la incorporación del ethos empresarial: más del 50\% de la matrícula está en el sector privado. Todo un logro, top one mundial" (Casassus, 2006: 5). En este estado de cosas tienen una gran responsabilidad los gobiernos de la concertación, aunque en estricto rigor, la situación actual es en gran medida el resultado de acciones permitidas, favorecidas y propiciadas por la actual configuración de fuerzas que conforman el "poder político" en Chile.

\section{LA GESTIÓN DEL SISTEMA EDUCATIVO}

Casassus plantea que en la gestión es posible identificar una secuencia de marcos conceptuales, técnicos e instrumentales, orientadores del cambio institucional, lo que se ha traducido en siete modelos de gestión: el normativo, el prospectivo, el estratégico, el estratégico situacional, calidad total, reingeniería y comunicacional. En esta trayectoria se ha transitado por distintas etapas de concreción o proceso de emergencia del sujeto. "En este proceso se tiene como sujeto en primer lugar al sistema en su conjunto, para luego dar lugar a la organización en sus distintos niveles administrativos, para luego terminar con las personas que constituyen la organización" (Casassus, 2005: 148). En esta "emergencia del sujeto humano", la gestión visibiliza a las personas como sujetos para el ejercicio del poder, entendido por Foucault como "una manera de actuar sobre un sujeto actuante o sobre sujetos actuantes, en tanto que actúan o son susceptibles de actuar. Un conjunto de acciones sobre otras acciones" (Foucault, 1988: 15). Así la gestión educativa se constituye en uno de los principales ámbitos e instrumentos para el ejercicio del poder en el sistema educativo, particularmente en el plano operativo, en el cual emergen los sujetos docentes, además, de los sujetos alumnos en su calidad de beneficiarios (o clientes) del sistema educativo.

En la reforma educativa chilena de los años 90, al igual que las demás reformas Latinoamericanas, la gestión se constituyó en el foco principal de las políticas educativas. En la declaración de Quito se plantea que: "es necesario producir una transformación profunda en la gestión educativa tradicional, que permita articular efectivamente la educación con las demandas económicas, políticas y culturales, rompiendo el aislamiento de las acciones educativas, transformando su contribución en una efectiva palanca de desarrollo económico, de la justa distribución de la riqueza y de la participación ciudadana" (PROMEDLAC IV 1991). Esta declaración, casi imperativa, en la que se insta explícitamente a romper el "aislamiento de las acciones educativas" con el propósito de contribuir efectivamente al desarrollo económico, es un abierto direccionamiento por la opción de una gestión educativa en función del desarrollo económico.

Existe una reconceptualización de la educación desde la economía, lo que se traduce en la práctica como una nueva ideología de la educación sobre la base de conceptos económicos. Este hecho provoca una tensión conceptual y valórica según argumenta Casassus (2005:155). Estos conceptos económicos no se corresponden con los saberes propios de los profesionales de la educación, por tanto, le resultan ajenos y en los 
hechos no estarían entendiendo la nueva terminología, o tal vez no la estarían aceptando. Esta divergencia tiene relación con la especificidad de la formación profesional de los docentes y los valores que tradicionalmente la sustentan, lo que entra en conflicto con las nuevas orientaciones valóricas que surgen del mundo económico. Debido a la escasa cercanía del lenguaje económico con el lenguaje pedagógico tradicionalmente manejado en la escuela, en el ámbito de la gestión se produce un problema de relacionamientos comunicacionales superpuesto a las relaciones de poder entre los planificadores y los docentes directivos encargados de la ejecución de las políticas en el nivel de la organización escolar. Este hecho estaría provocando, según Casassus, "el establecimiento de un sistema con mundos paralelos y superpuestos entre la política y la práctica" (2005: 155), lo que se traduciría en un importante efecto de simulación en la gestión de las políticas por parte de los docentes directivos, es decir, en la práctica no se aplicarían las políticas como lo pretenden los planificadores. Sin duda que este aspecto involucra, como diría Foucault una "situación estratégica compleja" y aquí se habría configurado un problema de relaciones de poder que no está siendo abordado adecuadamente por la gestión del sistema educativo chileno.

\section{EL ESTAMENTO DOCENTE EN EL CAMPO DE RELACIONES DE PODER}

Los profesores fueron directa e indirectamente afectados por la nueva configuración en el campo de fuerzas del sistema educativo durante el gobierno militar. "El centro del accionar gubernamental respecto al sector pasa a ser el control de los profesores y el disciplinamiento de la cultura escolar, así como tempranamente, modificaciones al currículo de historia y ciencias sociales de acuerdo con la nueva ideología en control del Estado" (Cox, 2003: 23). El poder de la dictadura se deja sentir con toda su potencia sobre los profesores. Una de las claras y directas manifestaciones del poder del régimen militar en tal sentido consistió en despojar del status de funcionarios públicos al cuerpo de profesores.

En el período inicial de la dictadura, la política estatal hacia los docentes se expresó en algunas medidas específicas (Núñez, 2003:474): La supresión de las escuelas normales y la entrega de la formación inicial de los docentes a Universidades públicas y privadas; disolución de las tradicionales organizaciones gremiales y la creación del Colegio de Profesores de Chile en 1974 con dirigentes designados hasta 1985 y con afiliación obligatoria; supresión de consejos de participación consultiva y ejecutiva en la administración de la educación pública; el encuadramiento de las remuneraciones en la escala general de la administración pública, en condiciones de desmedro respecto a otros sectores de profesionales comparables. Este poder político estatal se manifiesta en un marco de operación de mecanismos de vigilancia ideológica y política bajo condiciones de autocensura y amedrentamiento. "El poder en la vigilancia jerarquizada de las disciplinas no se tiene como se tiene una cosa, no se transfiere como una propiedad; funciona como una maquinaria" (Foucault, 2005: 182). Así, en tiempos de la dictadura, los docentes chilenos se encuentran inmersos en esta maquinaria o aparato productor de poder disciplinario, en una red de relaciones de poder que busca su disciplinamiento.

Desde los años ochenta se desarrollan procesos que involucran ciertas presiones de cambio que van a afectar a los docentes. Núñez plantea que la elevación general de los niveles de escolaridad y el acceso del conjunto de la población a la creciente información 
proporcionada por los modernos medios de comunicación, "se ha traducido en una erosión de la autoridad o liderazgo cultural y la suerte de monopolio de saberes de nivel intermedio que ejercían los docentes" (Núñez, 2003:473). También afirma la probabilidad de que la sensación de pérdida o amenaza por esta razón podría contribuir al descenso de la autoestima observada en los docentes y a la sensación de que no son dignamente valorados y tratados por la sociedad. Si concordamos con Foucault en que "no existe relación de poder sin constitución correlativa de un campo de saber, ni de saber que no suponga y no constituya al mismo tiempo unas relaciones de poder" (Foucault, 2005: 34), podemos considerar que el acceso creciente de gran parte de la población a nuevos saberes reconfigura un nuevo campo de distribución de saberes, que lleva consecuentemente aparejado un cambio en la distribución del poder, es decir, por esta causa también se afecta la configuración dinámica de las relaciones de poder en la sociedad en general $\mathrm{y}$ en el sistema educativo en particular.

Respecto de los intereses de las reformas educativas en relación con los docentes, Beatriz Ávalos plantea que "la finalidad perseguida es el logro de resultados que, en general, se refieren a cambios en los alumnos y alumnas, definidos principalmente, hoy día, como los que miden los instrumentos nacionales y o internacionales. Este esquema algo simplístico se refiere a un concepto de reforma diseñado y dirigido centralmente" (Ávalos, 2003: 564). El desempeño profesional docente en Chile está relacionado directamente con un enfoque de gestión escolar que pretende centrarse en la calidad, noción que aunque no se encuentra explícitamente definida, habría que referirla a logros de aprendizaje de los alumnos, a juzgar por el énfasis puesto en las mediciones nacionales. El problema de la calidad se focaliza en la práctica de los docentes, debido a los cambios poco significativos en los aprendizajes que las mediciones SIMCE han puesto en evidencia. Al respecto Cox concluye: "El problema de la calidad hoy se concentra en la enseñanza en el aula, y en la necesidad de incrementar radicalmente la eficacia de los apoyos para su mejoramiento, así como de resolver el déficit de preparación de los docentes para enseñar el nuevo currículum" (Cox, 2003: 101).

Los docentes pasan a ocupar el centro de atención y se ejerce un cuestionamiento público respecto de la calidad de su desempeño profesional. Así, adquieren la visibilidad necesaria para una nueva manifestación del poder disciplinario. A partir del año 2003, se originó un Sistema de Evaluación del Desempeño Docente Profesional, dirigido a los docentes de aula del sistema municipal, a partir de Acuerdo Marco tripartito suscrito por el Ministerio de Educación, la Asociación Chilena de Municipalidades y el Colegio de Profesores de Chile, acuerdo que se plasmó en las leyes 19.933 y 19.961. Los criterios de evaluación están establecidos en el "marco para la buena enseñanza". Este marco presenta cuatro dominios: preparación de la enseñanza, creación de un ambiente propicio para el aprendizaje, responsabilidades profesionales y enseñanza para el aprendizaje de todos los alumnos; 20 criterios del ejercicio profesional docente ordenados en los cuatro dominios; y un total de 70 descriptores para la evaluación del desempeño docente. Esta detallada estructura normativa nos evoca el análisis de Foucault (2005: 143) respecto de la disciplina como anatomía política del detalle.

Las reformas educativas reconocen el rol que tienen los docentes en su implementación y, por tanto, se preocupan de realizar actividades dirigidas a ellos: “a) dar a conocer la reforma; b) conseguir su apoyo; c) promover el aprendizaje de los nuevos conceptos o contenidos y d) ensayar las nuevas estrategias" (Ávalos, 2003: 564). Estas actividades que 
reflejan los intereses de la reforma educativa, permiten apreciar con claridad el rol que se espera de los docentes chilenos. Los profesores son importantes en tanto sirven como instrumentos de implementación de las reformas, deben ser los operadores de los cambios diseñados, dirigidos e impuestos centralizadamente. La gestión educativa se transforma en el instrumento de poder que desarrolla acciones directivas para estructurar el campo de las acciones posibles de los docentes. Aunque en tal contexto, los docentes no tienen participación, en el diseño de los cambios educativos y en la gestión de las políticas, es paradojal que en el momento de examinar responsabilidades por los malos resultados, prácticamente se exime de responsabilidad a los niveles de planeamiento y gestión. Así se desvía la atención de las variables estructurales hacia las variables individuales y se concentra en el docente toda la carga y tensión por los malos resultados.

A pesar que la reforma del 90, a través de sus programas de mejoramiento introduce múltiples elementos y recursos materiales de apoyo orientados a mejorar las condiciones de la práctica docente, incluyendo la dimensión de perfeccionamiento, los profesores no habrían valorizado suficientemente el sustantivo mejoramiento en sus condiciones de trabajo. Al respecto, Belleï (2001) expone dos razones que explicarían ese hecho: la primera se refiere a la forma de comunicar las políticas de reforma y de persuadir a los profesores para participar e involucrarse en ellas. El énfasis y el foco ha estado en una orientación, casi exclusiva, del tipo de cambio requerido para mejorar los aprendizajes de los alumnos y no se ha presentado el mejoramiento de los recursos materiales e intelectuales de los profesores como un directo apoyo a su labor de enseñanza, en definitiva un apoyo al desempeño docente. Así, los profesores habrían percibido los cambios propuestos como una exigencia más bien autoritaria. "Cuesta ver en ello una oportunidad de desarrollo profesional y más bien se lo percibe como una actualización del mandato de la autoridad educacional que reclama obediencia en el nuevo escenario" (Belleï, 2001: 3). La segunda razón, según Belleï, sería que los dirigentes gremiales han demostrado una gran sensibilidad para los problemas laborales del sector, pero no han tenido una sensibilidad equivalente respecto de los contenidos del mejoramiento educativo que promueve el gobierno y su relevancia para la profesión docente. No obstante, reconoce que en los últimos años el Colegio de Profesores ha complementado su discurso reivindicacionista con propuestas y críticas en el orden sustantivo de la Política Educacional.

\section{CONCLUSIONES}

En el nivel político y técnico pareciera existir excesiva confianza en los poderes estructurales para la implementación de las reformas educativas, sin prestar suficiente atención a las "situaciones estratégicas" que se dan en la complejidad del sistema educativo. En no pocas ocasiones pareciera ignorarse o desestimarse el potencial de ciertas acciones, relaciones de poder y resistencias que, de modo a veces, silencioso, podrían estar afectando los equilibrios o desequilibrios en el campo de fuerzas del sistema educativo $\mathrm{y}$, en alguna medida, incidiendo en la efectividad de las políticas educativas.

Los modelos de reforma educativos implementados en Chile en la práctica son jerarquizados o descendentes y no han propiciado una auténtica participación de los docentes. Los procesos de cambio que las reformas pretenden desarrollar, específicamente en lo que dice relación con una práctica docente efectivamente orientada al logro de aprendizajes 
de los alumnos, requieren de una base de compromiso de los docentes que favorezca un desempeño profesional creativo necesario para facilitar profundos procesos de aprendizaje. Compromiso muy difícil, sino imposible, de concitar con una relación de poder vertical propiciada por una gestión educativa que considera a los docentes exclusivamente como operadores de cambios dirigidos e impuestos estructuralmente.

La administración del sistema educativo a nivel macro y micro debería prestar una mayor atención a las resistencias indefectiblemente asociadas a las relaciones de poder del sistema educativo. Si se tiene una concepción tradicional del poder asociado a las estructuras o instituciones, probablemente existirá la tendencia a despreciar el potencial de resistencia de las personas, particularmente de los sujetos docentes, no obstante, son los principales agentes del proceso educativo. En el aula, lugar en que los profesores están en una situación estrategia de privilegio, en lo que a relaciones de poder se refiere, se juega el éxito o el fracaso de la reforma educativa. Al parecer habría que buscar fórmulas de solución distintas, que no pasan por mayores apoyos técnicos o materiales en combinación con mandatos o exigencias directas e indirectas respecto del desempeño profesional docente, sino que por una auténtica valorización del rol, capacidades y potencialidades de acción o resistencia de los profesores en el proceso de instalación de reformas educativas.

Aunque sería imposible negar la importancia de las relaciones de poder en la sociedad en general y en los sistemas educativos en particular, pocas veces se tiene cabal comprensión de los efectos, implicancias, alcances, limitaciones y proyecciones asociadas a la incesante e inexorable dinámica de relaciones de poder en los sistemas educativos. En este impredecible campo de fuerzas, tampoco pareciera haberse dimensionado suficientemente el potencial desequilibrante que tienen o tendrían las entidades o actores sociales que hubieren aprehendido la vinculación poder-saber y sus implicancias estratégicas, particularmente quienes hayan alcanzado o logren alcanzar saberes específicos sobre las relaciones de poder contextualizadas en el sistema educativo. Lo más probable es que las entidades o actores sociales que logren este cometido estarán en una situación estratégica potencialmente distinta en el campo de las relaciones de poder.

\section{REFERENCIAS BIBLIOGRÁFICAS}

Ávalos, B. (2003). La formación de profesores y su desarrollo profesional. Prácticas innovadoras en busca de políticas. El caso de Chile. En Cox, C. (ed.), Políticas educacionales en el cambio de siglo (pp. 559-594). Santiago de Chile: Editorial Universitaria S.A.

Belleï, C. (2001). El talón de Aquiles de la reforma. Análisis sociológico de la política de los 90 hacia los docentes en Chile. Extraído el 3 de agosto, 2010 de http://www.opech.cl/bibliografico/ Doc_Docente/bellei_talon_de_aquiles_2001.pdf

Casassus, J. (2007). Una nota crítica acerca del proyecto de Ley General de Educación. Extraído el 3 de agosto, $2010 \mathrm{de}$ http://www.opech.cl/bibliografico/doc_movest/jcasassus_nota_critica.pdf

Casassus, J. (2005). Problemas de la Gestión Educativa en América Latina. En Courard, H. y Quintanilla, P. (Comp.), Cuadra, A., Ojeda, B. y Ossandón, C. (Eds.), Educación, Conocimiento y Nuevas Tecnologías (pp. 137-162) [cd-rom]. Santiago de Chile: Doctorado en Cultura y Educación en América Latina. Universidad ARCIS.

Castells, M. (2005). Globalización, Desarrollo y Democracia: Chile en el contexto mundial. Chile: Fondo de Cultura Económica Chile S.A. 
CHILE, MINISTERIO DE EDUCACIÓN. (2004). Marco para la Buena Enseñanza. Santiago de Chile: CPEIP-Ministerio de Educación.

CONFERENCIA Regional de Ministros de Educación y de Ministros Encargados de la Planificación Económica de los Estados Miembros de América Latina y del Caribe. (1979). Declaración de México. Extraído el 3 de agosto, 2010 de http://unesdoc.unesco.org/ images/0015/001599/159987S.pdf

Cox, C. (2003). Las políticas educacionales de Chile en las últimas dos décadas del siglo XX. En C. Cox, Políticas educacionales en el cambio de siglo (pp. 19-113). Santiago de Chile: Editorial Universitaria S.A.

Foucault, M. (1988). El sujeto y el poder. Revista Mexicana de Sociología, vol. 50, n. 3, 3-20.

Foucault, M. (1991). Historia de la Sexualidad I. La Voluntad de Saber. México D F: Siglo XXI.

Foucault, M. (2005). Vigilar y castigar. Nacimiento de la prisión. Madrid: Siglo XXI.

Laval, C. (2005). La Escuela no es una Empresa. En Courard, H. y Quintanilla, P. (comp.), Cuadra, A., Ojeda, B. y Ossandón, C. (Eds.), Educación, Conocimiento y Nuevas Tecnologías (pp. 68-123) [cd-rom]. Santiago de Chile: Doctorado en Cultura y Educación en América Latina. Universidad ARCIS.

Nuñez, I. (2003). El profesorado, su gremio y la reforma de los noventa: presiones de cambio y evolución de la cultura docente. En Cox, C. (Ed.), Políticas educacionales en el cambio de siglo (pp. 455-514). Santiago de Chile: Editorial Universitaria S.A.

Promedlac IV, Cuarta Reunión del Comité Regional Intergubernamental del Proyecto Principal de Educación en América Latina y el Caribe (1991). Declaración de Quito. Extraído el 03 de agosto, $2010 \mathrm{de} \mathrm{http://unesdoc.unesco.org/images/0009/000905/090515s.pdf \# page}=42$ 